\title{
Palladium/Imadazolium Salt Mediated Cyclisations for the Synthesis of Heterocyclic Compounds
}

\author{
William Kofie ${ }^{1,2^{*}}$, Stephen Caddick ${ }^{2,3}$ \\ ${ }^{1}$ Centre for Drug Design and Development, Department of Pharmaceutical Chemistry, Faculty of Pharmacy and \\ Pharmaceutical Sciences, Kwame Nkrumah University of Science and Technology, Kumasi, Ghana \\ ${ }^{2}$ Centre for Biomolecular Design and Drug Development, The Chemistry Laboratory, CPES, University of Sussex, \\ Brighton, UK \\ ${ }^{3}$ Department of Chemistry, University College London, London, UK \\ Email: "williamkofie@hotmail.com, "wkofie.pharm@knust.edu.gh
}

Received 11 August 2015; accepted 29 November 2015; published 2 December 2015

Copyright (@) 2015 by authors and Scientific Research Publishing Inc.

This work is licensed under the Creative Commons Attribution International License (CC BY).

http://creativecommons.org/licenses/by/4.0/

(c) (i) Open Access

\section{Abstract}

The use of intramolecular reactions involving palladium/imidazolium salts to synthesize heterocyclic compounds is described. Reactivity of phenyl, ethyl and methyl substituents leading to isolation of various isomeric products is also illustrated. Rearrangement of phenyl intermediates to furnish benzoxazoles is also mentioned.

\section{Keywords}

$N$-Heterocyclic Carbenes, Heterocyclic Systems, Imidazolium Salt, Intramolecular Cyclicsations, Phosphine Ligands

\section{Introduction}

Palladium mediated reactions play significant role in organic synthesis. Intramolecular Heck reactions in particular have become useful for the synthesis of carbocylic and heterocyclic rings [1] [2]. Conventional coupling methods require the use palladium phosphine complexes as catalyst for Heck reactions. The mechanism for these palladium catalyzed coupling reactions involves four key stages namely, i) oxidative addition of aryl halide to $\mathrm{Pd}(0)$; ii) intramolecular carbometallation; iii) $\beta$-hydride elimination and lastly iv) reductive elimination to regenerate the $\operatorname{Pd}(0)$ catalyst for the continuation of the catalytic cycle [3].

${ }^{*}$ Corresponding author. 
The choice of ligand is very important in these palladium catalyzed reactions. In recent times, the development of novel ligands for transition metal catalysis and the study of their mechanistic pathway have received greater attention [4] [5]. Sterically hindered phosphine ligands for palladium-catalysed reactions in particular, have improved greatly the applications of many metal mediated transformations [6].

Despite their wider application in palladium mediated synthesis, phosphine ligands do suffer limitations. This has necessitated the search for practical alternatives to phosphines as ligands in metal mediated cyclisation reactions [7].

$N$-heterocyclic carbenes (NHC) are now potential candidates as alternative ligands in transition metal catalyzed reactions due to their advantages over their conventional phosphine counterparts. They are known to be excellent donors and tend to form strong bonds with metals, giving them very good catalytic activity [7] [8].

NHCs have also found use in palladium catalysis. In complexes where NHC is coordinated to palladium (II), catalytic activity of these species have been examined and confirmed. In particular, catalytic properties of palladium-carbene complexes have been proven in amination, Heck and Suzuki reactions [9].

Hartwig and Nolan [10] [11], have reported that palladium-NHC complexes may be produced as intermediates when imidazolium salts are employed as additives in palladium catalyzed transformations. Although a full mechanistic rational is yet to be established, it is assumed that, the palladium and imidazolium salts generate either neutral or cationic palladium-carbene complexes in situ during the course of the metal catalytic cycle [12]. Nevertheless it can be appreciated that palladium-NHC complexes are proving valuable in synthetic organic chemistry [13].

We previously reported the successful use of Pd-carbene complexes in intramolecular Heck reactions involving aromatic chlorides. This indicates the effectiveness of these Pd-NHC complexes in metal catalyzed reactions, particularly involving the less reactive aryl chlorides, [14]. Improved yields were achieved with addition of tetrabutyl ammonium salts. These reactions require generation of the carbene in situ from imidazolium salts and subsequent completion of the catalytic cycle leading to the generation of heterocyclic compounds.

We describe herein our findings on palladium/imidazolium salt mediated protocols for intramolecular cyclisation reactions on to aryl iodides. Our studies have revealed that palladium/imidazolium salt catalysis can be used to synthesize a range of heterocyclic compounds from cheaper and readily available starting materials and precursors. Yields of these reactions are particularly good, and the products are free from contamination by phosphine related by-products, making them easily isolable.

\section{Experimental}

\subsection{Chemicals and Instruments}

Melting points were carried out on Gallenkemp melting point apparatus and are uncorrected. IR spectra were recorded on a Perkin Elmer transform instrument as thin film neat of a solution dissolved in nujol. All nmr spectra were recorded on Bruker Fourier transform instruments with frequencies quoted in mega Hertz recorded in all experimental data. All coupling constants ( $J$ values) were quoted in Hertz. Chemical shifts are reported upfields in parts per million. Mass spectra were recorded on Kratos or Fisons double focusing spectrometers. X-ray crystallography experiments were carried out using Enraf Nonius diffractometer. Thin layer chromatography (tlc) was performed using Merck Kieselgel precoated silica gel plates and visualized with UV light, vaporized iodine or potassium permanganate solution. Flash chromatography was carried out using Merck Kieselgel 230-400 mesh. Organic solvents were purified by distilling over drying agents. Throughout the experimental, imidazolium salt refers to the ligand 1,3-bis-(2,6-diisopropylpheynyl)imidazolium chloride.

\subsection{General Method for the Alkylation of Benzyl Alcohol}

A suspension of 2-iodobenzylalcohol (1.0 eq) and $\mathrm{NaH}(2.0 \mathrm{eq})$ in THF was stirred at room temperature for 30 min. Alkenyl halide (2.0 eq) was added and the reaction mixture stirred for a further $16 \mathrm{~h}$. The reaction mixture was then filtered through celite, washed with water and the aqueous layer extracted with $\mathrm{Et}_{2} \mathrm{O}$. The combined organic fractions were dried $\left(\mathrm{MgSO}_{4}\right)$, filtered and solvent removed in vacuo to give the product as an oil.

\subsection{General Method for the Synthesis of Phenylacrylamide}

To a solution of acyl chloride (1.0 eq) in $\mathrm{Et}_{2} \mathrm{O}$ at $0^{\circ} \mathrm{C}$ was added slowly 2-iodoaniline (2.0 eq) and the reaction 
mixture left to warm to room temperature for $2 \mathrm{~h}$. This was then quenched with $\mathrm{HCl}(0.5 \mathrm{M})$, washed with $\mathrm{NaHCO}_{3}$ solution followed by water and the aqueous layer extracted with $\mathrm{Et}_{2} \mathrm{O}$. The combined organic fractions were dried $\left(\mathrm{MgSO}_{4}\right)$, filtered and solvent removed in vacuo to give a crude sample which was purified by flash chromatography (Silica gel) [eluent-Pet:Et $\mathrm{t}_{2} \mathrm{O}, 20: 1$ - 5:1] to give the title compound as a solid.

\subsection{General Method for the Synthesis of Isochromene and Isochroman}

Iodobenzyl ether (1.0 eq), $\mathrm{Cs}_{2} \mathrm{CO}_{3}(1.5 \mathrm{eq}), \mathrm{Pd}_{2}(\mathrm{dba})_{3}(1 \mathrm{~mol} \%)$ and imidazolium salt (1 mol\%) were placed in a reaction vessel and purged with nitrogen under vacuum. $N, N$-dimethylacetamide was then added and the mixture heated at $140^{\circ} \mathrm{C}$ for $15 \mathrm{~h}$. The reaction mixture was allowed to cool to room temperature, dissolved in diethyl ether and filtered through celite. The filtrate was washed with water and aqueous layer extracted with diethyl ether. Combined organic fractions were dried $\left(\mathrm{MgSO}_{4}\right)$, filtered and solvent removed to give crude sample which was purified by flash chromatography (silica gel) [eluent-Pet: $\mathrm{Et}_{2} \mathrm{O}, 20: 1-10: 1$ ] to give the desired compound.

\subsection{General Method for the Synthesis of Benzoxazole}

Iodo-benzamide (1.0 eq), $\mathrm{Cs}_{2} \mathrm{CO}_{3}(1.5 \mathrm{eq}), \mathrm{Pd}_{2}(\mathrm{dba})_{3}(1 \mathrm{~mol} \%)$, and imidazolium salt (1 mol\%), were place in a reaction vessel and purged with nitrogen under vacuum. $N, N$-dimethylacetamide was then added and the mixture heated at $140^{\circ} \mathrm{C}$ for $10 \mathrm{~h}$. This was allowed to cool to room temperature, dissolved in diethyl ether and filtered through celite. The filtrate was washed with water and the aqueous layer extracted with ether. Combined organic fractions were dried $\left(\mathrm{MgSO}_{4}\right)$, filtered and solvent removed to give crude sample which was purified by flash chromatography (silica gel) [eluent-Pet:Et ${ }_{2} \mathrm{O}, 20: 1$ - 5:1] to give the desired compound.

\section{Spectral and Analytical Data}

\section{1-Allyloxymethyl-2-iodobenzene (3.1)}

$\mathrm{R}_{\mathrm{f}}$ Pet:EtOAc, 6:1 (0.8).

$v_{\max } \mathrm{Cm}^{-1}$ 3406, 3048, 2935, 1695, 1451, 1345, 1090, 1004, 915, 736.

$\delta_{\mathrm{H}}\left(300 \mathrm{MHz}, \mathrm{CDCl}_{3}\right) 7.72(1 \mathrm{H} \mathrm{d}, J=7.7, \mathrm{ArH}), 7.40(1 \mathrm{H}, \mathrm{d} 7.7, \mathrm{ArH}), 7.22(1 \mathrm{H}, \mathrm{dd}, J=7.7,7.3 \mathrm{ArH}) 6.87$

(1H, app. t, $J=7.7$, $\mathrm{ArH}), 5.90\left(1 \mathrm{H}\right.$, ddt $\left.J=17.1,10.3,6.7,-\mathrm{CH}_{2} \mathrm{OCH}_{2} \mathrm{CHCH}_{2}\right), 5.28(1 \mathrm{H}, \mathrm{d} J=10.3$, $\left.-\mathrm{CH}_{2} \mathrm{OCH}_{2} \mathrm{CHCH}_{2}\right), 5.12$, (1H, $\left.J=17.1,-\mathrm{CH}_{2} \mathrm{OCH}_{2} \mathrm{CHCH}_{2}\right), 4.4\left(2 \mathrm{H}, \mathrm{s},-\mathrm{CH}_{2} \mathrm{OCH}_{2} \mathrm{CHCH}_{2}\right) 4.02(2 \mathrm{H}, \mathrm{d} J=6.7$, $-\mathrm{CH}_{2} \mathrm{OCH}_{2} \mathrm{CHCH}_{2}$ ).

$\delta_{\mathrm{C}}\left(75 \mathrm{MHz}, \mathrm{CDCl}_{3}\right) 141.04(\mathbf{C}), 139.53(\mathbf{C H}), 134.13(\mathbf{C H}), 130.66(\mathbf{C H}), 129.87(\mathbf{C H}), 128.84(\mathbf{C H})$, 117.76 $\left(\mathrm{CH}_{2}\right), 98.15(\mathbf{C}), 72.07\left(\mathrm{CH}_{2}\right), 69.55\left(\mathrm{CH}_{2}\right)$.

$\mathbf{m} / \mathbf{z}$ (EI) 274 ( $\left.\mathrm{M}^{+}, 25 \%\right), 231\left(\mathrm{M}^{+}-\mathrm{C}_{3} \mathrm{H}_{5}, 63 \%\right) 217$ (100\%), 90 (75\%).

1-But-2-enyloxymethyl-2-iodobenzene (3.2)

$\mathrm{R}_{\mathrm{f}}$ Pet:Et ${ }_{2} \mathrm{O}, 5: 1$ (0.88).

$v_{\max } \mathrm{Cm}^{-1}$ 3021, 2920, 1562, 1438, 1350, 1092, 1090, 1005, 959, 743.

$\delta_{\mathrm{H}}\left(300 \mathrm{MHz}, \mathrm{CDCl}_{3}\right) 7.70(1 \mathrm{H} \mathrm{d}, J=7.9, \operatorname{ArH}), 7.33(1 \mathrm{H}, \mathrm{d}$ 8.2, $\mathrm{ArH}), 7.22(1 \mathrm{H}, \mathrm{dd}, J=7.7,7.3 \mathrm{ArH}) 6.83$

$(1 \mathrm{H}$, dd, $J=7.7,7.3 \mathrm{ArH}), 5.69\left(1 \mathrm{H}\right.$, dq $\left.J=15.2,6.0,-\mathrm{CH}_{2} \mathrm{OCH}_{2} \mathrm{CHCHCH}_{3}\right), 5.58(1 \mathrm{H}$, dt $J=15.2,6.0$ $\left.-\mathrm{CH}_{2} \mathrm{OCH}_{2} \mathrm{CHCHCH}_{3}\right), 4.36,\left(2 \mathrm{H}, \mathrm{s},-\mathrm{CH}_{2} \mathrm{OCH}_{2} \mathrm{CHCH}_{3}\right), 3.39\left(2 \mathrm{H}, \mathrm{d}, \mathrm{J}=5.8,\left(-\mathrm{CH}_{2} \mathrm{OCH}_{2} \mathrm{CHCHCH}_{3}\right), 1.63\right.$ (3H, d $J=6.0,-\mathrm{CH}_{2} \mathrm{OCH}_{2} \mathrm{CHCHCH}_{3}$ ).

$\delta_{\mathrm{C}}\left(75 \mathrm{MHz}, \mathrm{CDCl}_{3}\right) 141.15(\mathbf{C}), 139.51(\mathbf{C H}), 130.25(\mathbf{C H}), 129.16(\mathbf{C H}), 128.72(\mathbf{C H}), 127.81 \mathrm{~N}(\mathrm{CH})$, $127.08(\mathbf{C H}), 98.26(\mathbf{C}), 71.84(\mathbf{C}), 66.43\left(\mathrm{CH}_{2}\right), 18.33\left(\mathrm{CH}_{3}\right)$.

$\mathbf{m} / \mathbf{z}(\mathrm{EI}) 288\left(\mathrm{M}^{+}, 20 \%\right), 244\left(\mathrm{M}^{+}, 40 \%\right) 217\left(\mathrm{M}+-\mathrm{OC}_{4} \mathrm{H}_{7}, 100 \%\right), 36(60 \%)$.

1-Cynnamyloxymethyl-2-iodobenzene (3.3)

$\mathrm{R}_{\mathrm{f}}$ Pet:Et ${ }_{2} \mathrm{O}, 9: 1$ (0.55).

$v_{\max } \mathrm{Cm}^{-1}$ 3033, 2848, 1557, 1443, 1354, 1107, 1090, 960, 746, 687.

$\delta_{\mathrm{H}}\left(300 \mathrm{MHz}, \mathrm{CDCl}_{3}\right) 7.92(1 \mathrm{H} \mathrm{d}, J=7.5, \mathrm{ArH}), 7.60(1 \mathrm{H}, \mathrm{d} J=7.9, \operatorname{ArH}), 7.42(2 \mathrm{H}, \mathrm{dd}, J=7.5,7.4 \mathrm{ArH})$,

7.32-7.10 (4H, m, ArH), 7.07 (1H, apt. t $J=7.9 \mathrm{ArH}), 6.57$ (1H, d, $\left.J=16.0,-\mathrm{CH}_{2} \mathrm{OCH}_{2} \mathrm{CHCHPh}\right), 4.65$ (2H, s, $\left.-\mathrm{CH}_{2} \mathrm{OCH}_{2} \mathrm{CHCHPh}\right), 4.37$ (2H, d $\left.J=6.0,-\mathrm{CH}_{2} \mathrm{OCH}_{2} \mathrm{CHCHPh}\right)$.

$\delta_{\mathrm{C}}\left(75 \mathrm{MHz}, \mathrm{CDCl}_{3}\right) 141.108(\mathbf{C}), 139.68(\mathbf{C H}), 137.18(\mathbf{C}), 133.13(\mathbf{C H}), 129.71(\mathbf{C H}), 129.34(\mathbf{C H}), 129.10$ $(\mathbf{C H}), 128.76(\mathbf{C H}), 128.25(\mathbf{C H}), 127.07(\mathbf{C H}), 126.35(\mathbf{C H}), 98.47(\mathbf{C}), 76.43\left(\mathrm{CH}_{2}\right), 71.79\left(\mathrm{CH}_{2}\right)$. 
$\mathbf{m} / \mathbf{z}(\mathrm{ES}) 386\left(\mathrm{MNH}_{4}^{+}, 100 \%\right), 305$ (100\%).

$N$-(2-Iodophenyl)-3-phenylacrylamide (3.6)

$\mathrm{R}_{\mathrm{f}}$ Pet:EtOAc 6:1 (0.41).

Mp. $160^{\circ} \mathrm{C}-162^{\circ} \mathrm{C}$.

$v_{\max } \mathrm{Cm}^{-1} 3189,1655,1620,1451,1374,731$.

$\delta_{\mathrm{H}}\left(300 \mathrm{MHz}, \mathrm{CDCl}_{3}\right) 8.3(1 \mathrm{H}$, appt. d $J=7.5, \mathrm{NH}), 7.74-7.72(1 \mathrm{H}, \mathrm{m}, \operatorname{ArH}), 7.68(1 \mathrm{H}, \mathrm{d} J=15.8$, -COCHCH-), 7.56-7.49 (3H, m, ArH), 7.36-7.18 (4H, m, ArH), 6.79 (1H, appt. t $J=7.9$, ArH), $6.52(1 \mathrm{H}, \mathrm{d} J=$ 15.5 -СOCHCH-).

$\delta_{\mathrm{C}}\left(75 \mathrm{MHz}, \mathrm{CDCl}_{3}\right) 164.30(\mathbf{C}=\mathrm{O}), 143.41(\mathbf{C}), 139.24(\mathbf{C}), 138.70(\mathbf{C H}), 134.82(\mathbf{C H}), 130.60(\mathbf{C H}), 129.75$ (CH), $129.32(\mathrm{CH}), 128.50(\mathrm{CH}), 126.44(\mathrm{CH}), 122.46(\mathrm{CH}), 120.98(\mathrm{CH}), 90.05(\mathrm{C})$.

$\mathbf{m} / \mathbf{z}(\mathrm{EI}) 349\left(\mathrm{M}^{+}, 30 \%\right), 222\left(\mathrm{M}^{+}-\mathrm{I}, 80 \%\right), 131\left(\mathrm{M}^{+}-\mathrm{C}_{6} \mathrm{H}_{5} \mathrm{NI}, 100 \%\right)$.

$\mathrm{N}$-(2-Iodophenyl)-benzamide (3.7)

$\mathrm{R}_{\mathrm{f}}$ Pet:Et ${ }_{2} \mathrm{O} 6: 1(0.39)$.

Mp. $140^{\circ} \mathrm{C}-142^{\circ} \mathrm{C}$.

$v_{\max } \mathrm{Cm}^{-1} 3214,1639,1506,1456,1373,1287,739,704$.

$\delta_{\mathrm{H}}\left(300 \mathrm{MHz}, \mathrm{CDCl}_{3}\right) 8.40(1 \mathrm{H}, \mathrm{d} J=8.2, \mathrm{ArH}), 8.22(1 \mathrm{H}, \mathrm{s}, \mathrm{NH}), 7.90(2 \mathrm{H}, \mathrm{dd}, J=8.2,7.9, \mathrm{ArH}), 7.74(1 \mathrm{H}$,

d $J=7.9$, ArH), 7.56-7.40 (3H, m, ArH), 7.34 (1H, dd, $J=8.2,7.3$, ArH), 6.81 (1H, dd, $J=7.5,7.7$, ArH).

$\delta_{\mathrm{C}}\left(75 \mathrm{MHz}, \mathrm{CDCl}_{3}\right) 165.70(\mathbf{C}=\mathrm{O}), 139.21(\mathbf{C}), 138.66(\mathbf{C}), 134.92(\mathbf{C H}), 132.60(\mathbf{C H}), 130.57(\mathbf{C H}), 129.83$

(CH), $129.36(\mathbf{C H}), 127.57(\mathbf{C H}), 126.44(\mathbf{C H}), 122.14(\mathbf{C H}), 90.59(\mathbf{C})$.

$\mathbf{m} / \mathbf{z}(\mathrm{EI}) 323$ (M+1, 10\%), 196 ( $\left.\mathrm{M}^{+}-\mathrm{I}, 62 \%\right), 122$ (87\%), $105\left(\mathrm{M}^{+}-\mathrm{C}_{6} \mathrm{H}_{5} \mathrm{NI}, 100 \%\right)$.

4-Methyl-1H-isochromene (4.1)

$\mathrm{R}_{\mathrm{f}}$ Pet:Et $_{2} \mathrm{O}, 9: 1$ (0.87).

$v_{\max } \mathrm{Cm}^{-1}$ 3048, 2844, 1640, 1484, 1354, 1448, 1140, 1107, 1007, 936, 72.

$\delta_{\mathrm{H}}\left(300 \mathrm{MHz}, \mathrm{CDCl}_{3}\right) 7.18,(1 \mathrm{H} \mathrm{d}, J=7.5, \operatorname{ArH~}), 7.07(1 \mathrm{H} \mathrm{dd} J=7.5,7.4 \mathrm{ArH}), 7.01(1 \mathrm{H}, \mathrm{d}, J=7.5 \mathrm{ArH})$,

6.93 (1H, dd $J=7.5,7.3$, ArH), 6.39 (1H, s, - $\left.\mathrm{CH}_{2} \mathrm{OCHC}-\mathrm{Me}\right), 4.91$ (2H, s, $\left.-\mathrm{CH}_{2} \mathrm{OCH}-\right), 1.84$ (3H, s, $\mathrm{CH}_{3}$ ).

$\delta_{\mathrm{C}}\left(75 \mathrm{MHz}, \mathrm{CDCl}_{3}\right) 141.28(\mathbf{C H}), 131.34(\mathbf{C H}), 127.68(\mathbf{C}), 127.02(\mathbf{C H}), 125.61(\mathbf{C H}), 122.68(\mathbf{C H})$,

$119.26(\mathbf{C H}), 110.36(\mathbf{C}), 67.20\left(\mathrm{CH}_{2}\right), 12.07\left(\mathrm{CH}_{3}\right)$.

$\mathrm{m} / \mathrm{z}$ (EI) 146 (M+1, 85\%), 117 (100\%), 91 (15\%).

4-Ethyl-1H-isochromene (4.2)

$\mathrm{R}_{\mathrm{f}}$ Pet:Et $2 \mathrm{O}, 10: 1(0.88)$.

$v_{\max } \mathrm{Cm}^{-1}$ 3062, 2960, 2834, 1628, 1483, 1448, 1139, 1107, 935, 747.

$\delta_{\mathrm{H}}\left(300 \mathrm{MHz}, \mathrm{CDCl}_{3}\right) 7.18,(1 \mathrm{H} \mathrm{d}, J=7.0, \operatorname{ArH~}), 7.09$ (2H dd $\left.J=7.7,7.3 \mathrm{ArH}\right), 6.95(1 \mathrm{H}, \mathrm{d}, J=7.9 \operatorname{ArH})$,

$6.40\left(1 \mathrm{H}, \mathrm{s},-\mathrm{CH}_{2} \mathrm{OCHCCH} \mathrm{CH}_{3}\right), 4.89\left(2 \mathrm{H}, \mathrm{s},-\mathrm{CH}_{2} \mathrm{OCHCCH}_{2} \mathrm{CH}_{3}\right), 2.31\left(2 \mathrm{H}, \mathrm{q} J=7.5,-\mathrm{CH}_{2} \mathrm{OCHCCH}_{2} \mathrm{CH}_{3}\right)$

$1.09\left(3 \mathrm{H}, \mathrm{t} J=7.5,-\mathrm{CH}_{2} \mathrm{OCHCCH}_{2} \mathrm{CH}_{3}\right)$.

$\delta_{\mathrm{C}}\left(75 \mathrm{MHz}, \mathrm{CDCl}_{3}\right) 142.26(\mathbf{C H}), 131.92(2 \mathrm{XC}), 129.41(\mathbf{C H}), 128.39(\mathbf{C H}), 126.87(\mathbf{C H}), 124.36(\mathbf{C H})$,

$120.58(\mathbf{C H}), 118.10(\mathbf{C}), 68.65\left(\mathrm{CH}_{2}\right), 21.14\left(\mathrm{CH}_{2}\right), 13.92\left(\mathrm{CH}_{3}\right)$.

$\mathrm{m} / \mathrm{z}$ (EI) 160 (M+1, 85\%), 117 (100\%), 91 (55\%), 49 (80\%).

4-Benzyl-1H-isochromene (4.3)

$\mathrm{R}_{\mathrm{f}}$ Pet:Et $2 \mathrm{O}$ 9:1 (0.8).

Mp. $53^{\circ} \mathrm{C}-55^{\circ} \mathrm{C}$.

$v_{\max } \mathrm{Cm}^{-1} 29684,1623,1454,1448,1141,956$.

$\delta_{\mathrm{H}}\left(300 \mathrm{MHz}, \mathrm{CDCl}_{3}\right) 7.27,(1 \mathrm{H} \mathrm{d}, J=7.0, \mathrm{ArH}), 7.22-7.19$ (3H, m, ArH), 7.08-7.05 (3H, m, ArH), 6.95 (2H, appt. t, $J=7.7 \mathrm{ArH}), 6.40\left(1 \mathrm{H}, \mathrm{s},-\mathrm{CH}_{2} \mathrm{OCHC}-\right), 4.96\left(2 \mathrm{H}, \mathrm{s},-\mathrm{CH}_{2} \mathrm{OCHCC}-\right), 3.61\left(2 \mathrm{H}, \mathrm{s}-\mathrm{CH}_{2} \mathrm{Ph}\right)$.

$\delta_{\mathrm{C}}\left(75 \mathrm{MHz}, \mathrm{CDCl}_{3}\right) 144.79(\mathbf{C H}), 140.02(\mathbf{C}), 131.74(2 \mathrm{XC}), 129.16(\mathbf{C H}), 128.81(2 \times \mathbf{C H}), 128.78(\mathbf{C H})$,

128.42 (CH), $127.07(\mathbf{C H}), 126.58(\mathbf{C H}), 124.29(\mathbf{C H}), 121.31(\mathbf{C H}), 115.10(\mathbf{C}), 68.81\left(\mathrm{CH}_{2}\right), 34.36\left(\mathrm{CH}_{2}\right)$.

$\mathbf{m} / \mathbf{z}$ (EI) 222 ( $\left.\mathrm{M}^{+}, 55 \%\right), 57$ (57\%), 43 (100\%), 28 (60\%).

4-Benzylidene-isochroman (4.4)

$\mathrm{R}_{\mathrm{f}}$ Pet:Et ${ }_{2} \mathrm{O}$ 9:1 (0.5).

Mp. $78^{\circ} \mathrm{C}-80^{\circ} \mathrm{C}$.

Elemental Analysis: Expected; C 86.45\%, H 6.35\%, O 7.20\%.

Found; C 86.62\%, H 6.24\%, O 7.14.

$v_{\max } \mathrm{Cm}^{-1}$ 3379, 2983, 1712, 1596, 1492, 1447, 1268, 1100, 764.

$\delta_{\mathrm{H}}\left(300 \mathrm{MHz}, \mathrm{CDCl}_{3}\right) 7.66(1 \mathrm{H}, \mathrm{d} J=7.3, \operatorname{ArH}), 7.27,(2 \mathrm{H}$ appt t, $J=7.5, \operatorname{ArH}), 7.19-7.12(5 \mathrm{H}, \mathrm{m}, \operatorname{ArH})$, 
7.08 (1H, s, $\left.\left.-\mathrm{CH}_{2} \mathrm{OCH}_{2} \mathrm{CCH}-\mathrm{Ph}\right), \mathrm{ArH}\right), 6.95$ (1H, d, $\left.J=7.3 \mathrm{ArH}\right), 4.68$ (2H, s, - $\left.\mathrm{CH}_{2} \mathrm{OCH}_{2} \mathrm{CCH}-\mathrm{Ph}\right), 4.66$ (2H, d $\left.J=1.5-\mathrm{CH}_{2} \mathrm{OCH} \mathbf{H}_{2} \mathrm{CCH}-\mathrm{Ph}\right)$.

$\delta_{\mathrm{C}}\left(75 \mathrm{MHz}, \mathrm{CDCl}_{3}\right) 137.09(\mathbf{C}), 135.27(\mathbf{C}), 132.68(\mathrm{C}), 132.57(\mathrm{C}), 129.72(\mathbf{C H}), 128.78(\mathbf{C H}), 128.04$ $(\mathbf{C H}), 127.60(2 \times \mathbf{C H}), 127.59(2 \times \mathbf{C H}), 126.58(\mathbf{C H}), 125.15(\mathrm{CH}), 123.81(\mathrm{CH}), 123.66(\mathbf{C H}), 69.01\left(\mathrm{CH}_{2}\right)$, $67.28\left(\mathrm{CH}_{2}\right)$.

$\mathbf{m} / \mathbf{z}(\mathrm{EI}) 222\left(\mathrm{M}^{+}, 100 \%\right), 178$ (60\%), 115 (95\%).

2-Styryl-benzooxazole (4.5)

$\mathrm{R}_{\mathrm{f}}$ Pet:EtOAc 3:1 (0.7).

Mp. $90^{\circ} \mathrm{C}-92^{\circ} \mathrm{C}$.

$v_{\max } \mathrm{Cm}^{-1} 2984,1637,1530,1453,1372$.

$\delta_{\mathrm{H}}\left(300 \mathrm{MHz}, \mathrm{CDCl}_{3}\right) 7.81(1 \mathrm{H}, \mathrm{d} J=16.3$, -CHCHPh), $7.74(1 \mathrm{H}, \mathrm{d} J=7.5 \mathrm{ArH}) 7.73(2 \mathrm{H}, \mathrm{dd} J=7.6,7.4$, ArH), 7.54 (1H, d $J=7.5 \mathrm{ArH}), 7.43$ (2H, dd $J=7.6,7.6 \mathrm{ArH}), 7.36-7.34$ (3H, m ArH), 7.10 (1H, d $J=16.3$ $\mathrm{CHCHPh).}$

$\delta_{\mathrm{C}}\left(75 \mathrm{MHz}, \mathrm{CDCl}_{3}\right) 161.76(-\mathrm{NC}), 149.37(\mathbf{C}), 141.15(\mathbf{C}), 138.42(\mathbf{C H}), 134.1(\mathbf{C}), 128.74(\mathbf{C H}), 127.94$ $(\mathbf{C H}), 127.33(\mathbf{C H}), 126.52(\mathbf{C H}), 126.20(\mathbf{C H}), 124.18(\mathbf{C H}), 123.47(\mathbf{C H}), 118.84(\mathbf{C H}), 112.91(\mathbf{C H}), 101.29$ (CH).

m/z (EI) 221 (M+1, 80\%), $220\left(\mathrm{M}^{+}\right.$- H 100\%), 191 (45\%).

\section{2-Phenyl-benzooxazole (4.6)}

$\mathrm{R}_{\mathrm{f}}$ Pet:EtOAc 6:1 (0.81).

Mp. $110^{\circ} \mathrm{C}-112^{\circ} \mathrm{C}$.

$v_{\max } \mathrm{Cm}^{-1} 2986,1611,1550,1458,1373,1233,1045,731$.

$\delta_{\mathrm{H}}\left(300 \mathrm{MHz}, \mathrm{CDCl}_{3}\right)$ 8.22-8.17, $(2 \mathrm{H}, \mathrm{m}, \mathrm{ArH}), 7.72-7.69(1 \mathrm{H}, \mathrm{m}, \mathrm{ArH}), 7.47-7.44(4 \mathrm{H}, \mathrm{m}, \mathrm{ArH}), 7.30-7.27$

(2H, m, ArH).

$\delta_{\mathrm{C}}\left(75 \mathrm{MHz}, \mathrm{CDCl}_{3}\right) 149.73(\mathbf{C}), 141.07(\mathbf{C}), 130.49(\mathbf{C}), 127.89(2 \times \mathbf{C H}), 126.59(2 \times \mathbf{C H}), 126.13(\mathbf{C})$, 126.20 (CH), $124.08(\mathbf{C H}), 123.55(\mathbf{C H}), 118.99(\mathbf{C H}), 109.57(\mathbf{C H})$.

m/z (EI) 195 (M+1 100\%), 167 (50\%), 77 (40\%), 63 (50\%).

\section{Results and Discussion}

Starting materials were obtained by standard synthetic transformations, where 2-iodobenzylalcohol was deprotonated and the anion subsequently quenched with the corresponding alkenylbromide [15] [16], to furnish the desired alkenyloxymethyl-2-iodobenzene as the cyclisation precursors, 3.1-3.3 (Scheme 1). To obtain oxyindoles as final heterocyclic products, acrylamide precursors were synthesized by direct acylation of iodoaniline with enoyl chlorides to give desired products 3.4-3.6 (Scheme 2), [17].

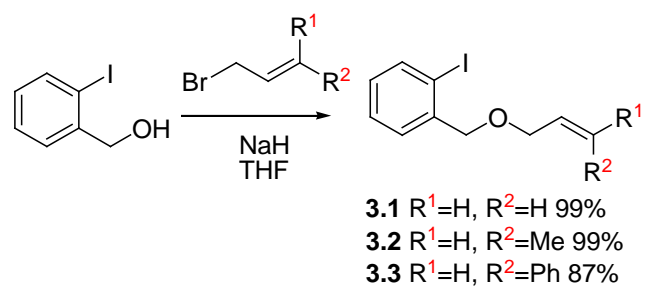

Scheme 1. Synthesis of benzyloxy precursors used in the synthesis of bicyclic rings.

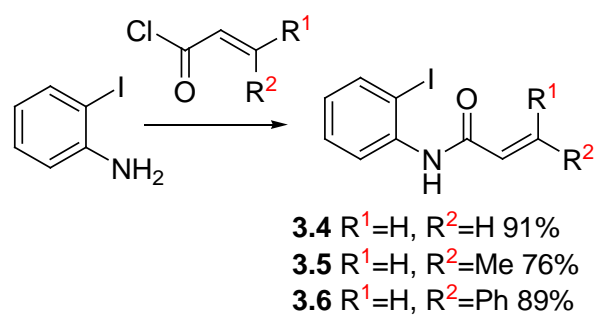

Scheme 2. Synthesis of amide precursors which gave oxazoles upon cyclisations. 
When alkenyloxy precursors $\mathbf{3 . 1}$ and $\mathbf{3 . 2}$ were subjected to reaction conditions using the palladium/imidazolium salt protocol, the desired bicyclic isochromene products $\mathbf{4 . 1}$ and $\mathbf{4 . 2}$ were isolated in very good yields following purification by column chromatography. The cyclisations proceeded possibly via 6-exo fashion followed by rapid isomerization to give the products obtained, (Scheme 3 ).
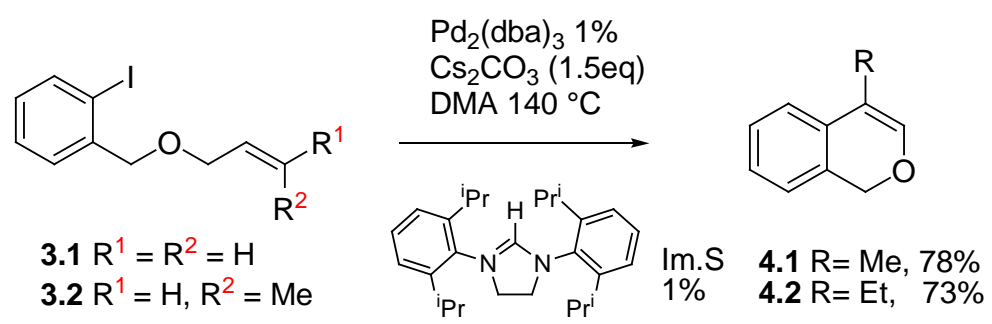

Scheme 3. Pd/ Im.S mediated cyclisations to produce bicyclic ring systems (Im.S = imidazolium salt).

When the phenyl substituted precursor 3.3 was subjected to the same reaction conditions, however, two isomeric products were isolated [18]. Benzylisochromene 4.3 [19], and benzylideneisochroman 4.4 [20] [21], nuclei were obtained in yields of 22\% and 63\% respectively, (Scheme 4).

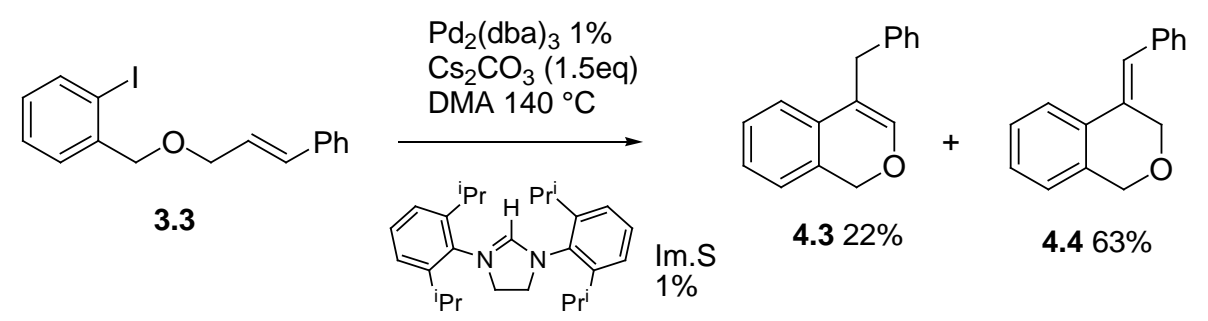

Scheme 4. Phenyl substituted precursor gave isomers $\mathbf{4 . 3}$ and $\mathbf{4 . 4}$ when subjected to $\mathrm{Pd} /$ Im.S conditions.

Isolation of benzylideneisochroman $\mathbf{4 . 4}$ as the major product in this case means the reaction probably proceeded via 6-exo cyclisation. Unlike the previous examples where isomerization is presumed to have taken place following cyclisation to give the kinetically stable products, benzylideneisochroman 4.4 (Figure 1) happen to be the major isolated product from cyclisation of 1-cynnamyloxymethyl-2-iodobenzene, 3.3. Although there is a possible 1,3-hydrogen shift to generate isochromene 4.3, the isolation of isochroman $\mathbf{4 . 4}$ as the major product may be due to the conjugation system that extends from the alkene bond onto the phenyl ring to give a more stable isomer.

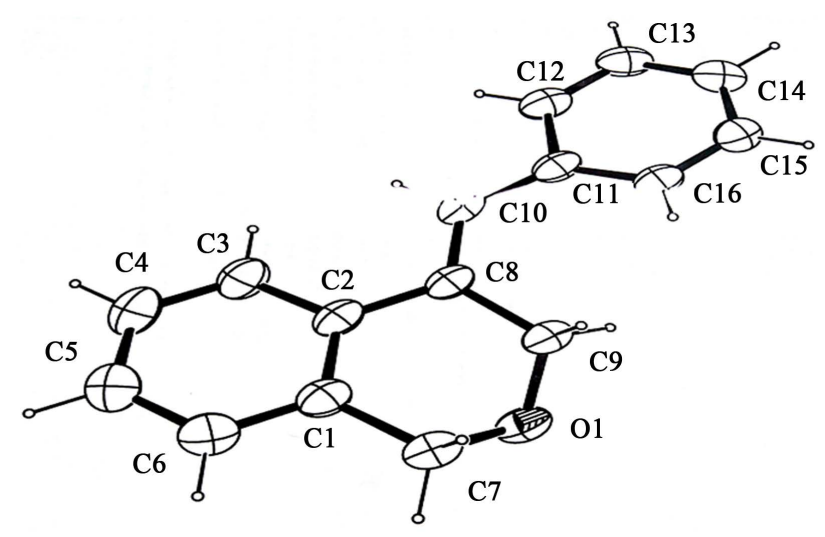

Figure 1. Crystal structure of benzylideneisochroman 4.4, major isomer from phenyl substituted precursor. 
It was disappointing to observe that, treating but-2-enoic phenyl amide and phenyl acrylamide substrates with palladium/imidazolium salt failed to give the desired oxyindoles. Surprisingly, what seemed to be loss of the alkenoyl moiety leading to isolation of 2-iodoaniline was observed, (Scheme 5).

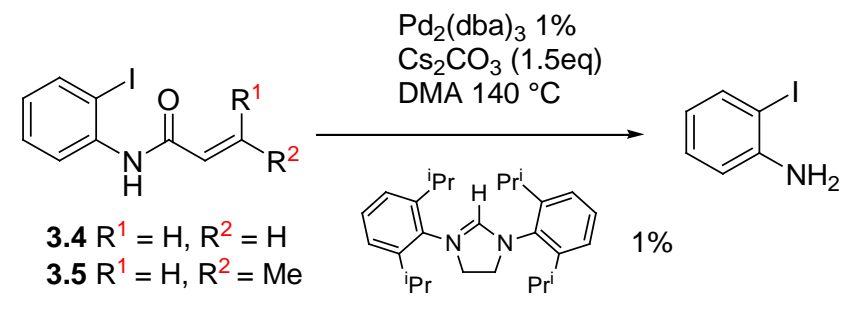

Scheme 5. Reaction of alkenoyl moiety upon treatment with Pd/Im.S.

When $N$-(2-iodophenyl)-3-phenylacylamide 3.6 was subjected to palladium/imidazolium salt protocols, there was conversion of starting material to product after heating for $3 \mathrm{~h}$. It was revealed by spectral analysis that the desired oxyindole had not been formed, instead cyclisation had occur to give 2-styryl-benzooxazole 4.5, [22] [23], (Scheme 6), (Figure 2) [24].

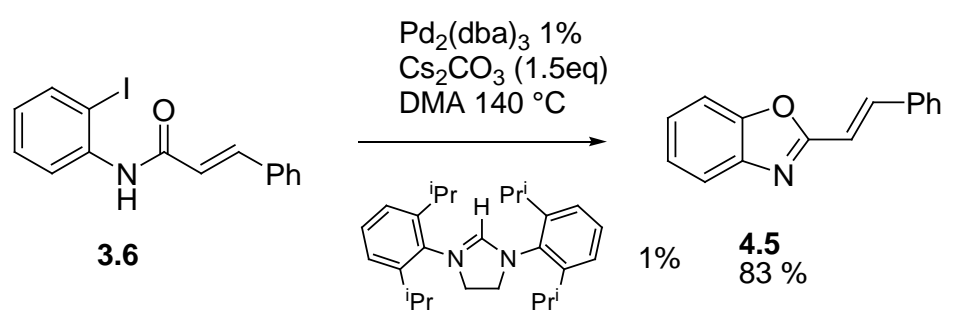

Scheme 6. Cyclisation to give 2-styryl-benzooxazole using Pd/Im.S.

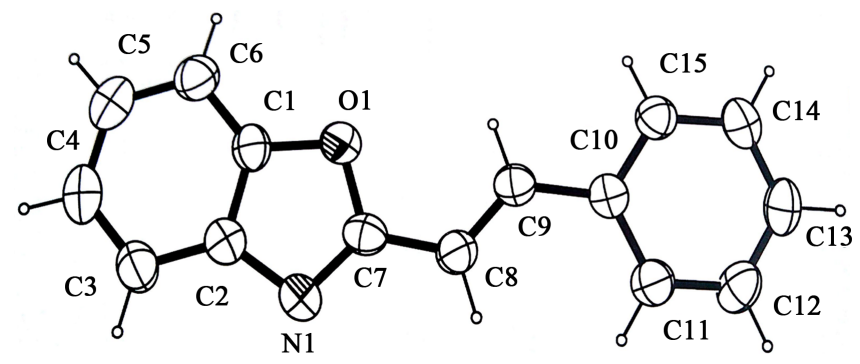

Figure 2. Crystal structure of 2-styryl-benzooxazole, 4.5 .

To further extend the scope of these findings, $N$-(2-iodophenyl)benzamide 3.7 and $N$-(2-iodophenyl) acetamide were subjected to the same reaction conditions. While the benzamide reacted possibly via o-arylation to produce 2-phenylbenzooxazole $\mathbf{4 . 6}$ in good yield, no product was isolated when the acetamide derivative was subjected to the same reaction conditions, (Scheme 7).

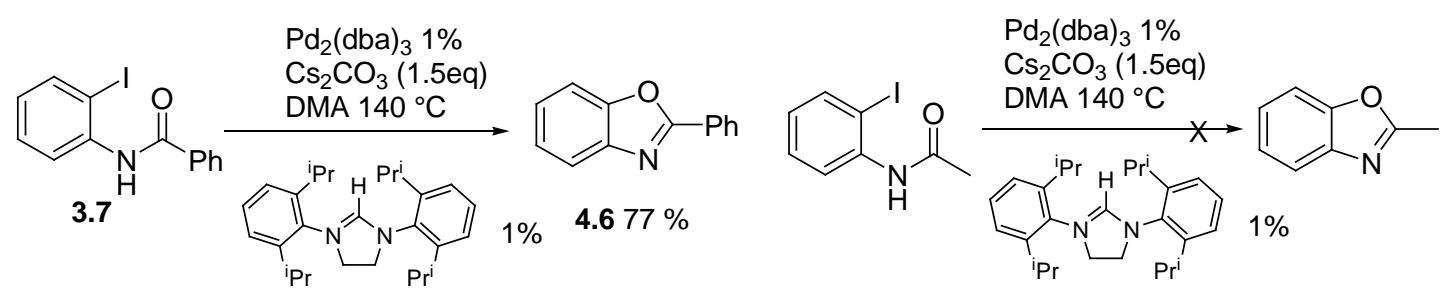

Scheme 7. Under Pd/Im.S condition, iodophenylbenzamide gave Phenyl benzooxazole, 4.6 whiles the acetamide intermediate gave no desired product. 
We believe the ability of the phenyl substrates to efficiently undergo cyclisation may be due to the phenyl group providing strong steric and electronic effects leading to a stable rotamer with a configuration that allows the carbonyl oxygen to be within close proximity to the iodine, and thus favouring an attack on to the ring, (Scheme 8).

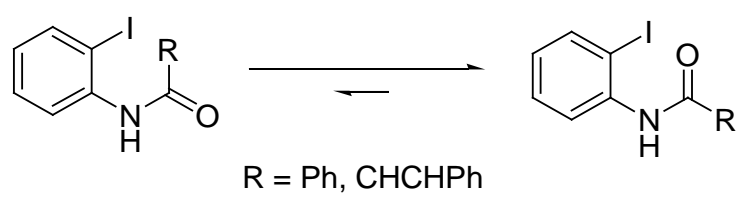

Scheme 8. Rotation around the carbonyl showing a shift in equilibrium to the more favourable rotamer.

\section{Conclusion}

In conclusion, we have shown that palladium imidazolium salt protocols can be used to promote intramolecular cyclisation reactions, enabling the synthesis of fused bicyclic heteroaromatics with great efficiency. Phenyl substituents have strong influence on reaction outcomes as a result of steric and electronic factors. Iodophenylbenzamide substrates undergo cyclisation to give substituted benzooxazoles. The ease with which phenyl containing amides undergo cyclisation to produce substituted benzooxazoles is noteworthy.

\section{Acknowledgements}

We gratefully acknowledge Dr. John Hayler at GSK Tonbridge for his encouragement, support and helpful comments throughout this project. We also gratefully acknowledge support of this project from GSK and EPSRC. We are grateful to EPSRC Mass Spectrometry Service at Swansea and Drs Abdul-Sada, Avent and Hitchcock of The University of Sussex for their contributions to this work. We gratefully acknowledge the support by members of staff of department of pharmaceutical chemistry KNUST, Kumasi Ghana. We are also grateful to Lagray Chemicals, Ghana Limited for their regular support.

\section{References}

[1] Belestkaya, I.P. and Cheprakov, A.V. (2000) The Heck Reaction as a Sharpening Stone of Palladium Catalysis. Chemical Reviews, 100, 3009-3066. http://dx.doi.org/10.1021/cr9903048

[2] Oestreich, M., Dennison, P.R., Kodanko, J.J. and Overman, L.E. (2001) Thwarting $\beta$-Hydride Elimination: Capture of the Alkylpalladium Intermediate of an Asymmetric Intramolecular Heck Reaction. Angewandte Chemie International Edition, 40, 1439-1442. http://dx.doi.org/10.1002/1521-3773(20010417)40:8<1439::AID-ANIE1439>3.0.CO;2-F

[3] Tsuji, J. (1997) Palladium Reagents and Catalyst; Innovations in Organic in Organic Synthesis. Wiley \& Sons.

[4] Alcazar-Roman, L.M. and Hartwig, J.F. (2001) Mechanism of Aryl Chloride Amination: Base-Induced Oxidative Addition. Journal of the American Chemical Society, 123, 12905-12906. http://dx.doi.org/10.1021/ja016491k

[5] Alcazar-Roman, L.M. and Hartwig, J.F. (2002) Mechanistic Studies on Oxidative Addition of Aryl Halides and Triflates to $\mathrm{Pd}(\mathrm{BINAP})_{2}$ and Structural Characterization of the Product from Aryl Triflate Addition in the Presence of Amine. Organometallics, 21, 491-502. http://dx.doi.org/10.1021/om0108088

[6] Muci, A.R. and Buchwald, S.L. (2002) Practical Palladium Catalysts for C-N and C-O Bond Formation. Topics in Current Chemistry, 219, 131-201. http://dx.doi.org/10.1007/3-540-45313-X_5

[7] Northeton, M.R. and Fu, G.C. (2001) Air-Stable Trialkylphosphonium Salts: Simple, Practical, and Versatile Replacements for Air-Sensitive Trialkylphosphines. Applications in Stoichiometric and Catalytic Processes. Organic Letters, 3 , 4295-4298. http://dx.doi.org/10.1021/ol016971g

[8] Herrmann, W.A., Weskamp, T. and Bohm, V.P.W. (2002) Metal Complexes of Stable Carbenes. Advances in Organometallic Chemistry, 48, 1-69. http://dx.doi.org/10.1016/S0065-3055(01)48001-4

[9] Böhm, V.P.W., Gstöttmayr, C.W.K., Westkamp, T. and Herman, W.A. (2000) N-Heterocyclic Carbenes: Part 26. $\mathrm{N}$-Heterocyclic Carbene Complexes of Palladium(0): Synthesis and Application in the Suzuki Cross-Coupling Reaction. Journal of Organometallic Chemistry, 596, 186-190. http://dx.doi.org/10.1016/S0022-328X(99)00590-2

[10] Staueeer, S.R., Lee, S., Sambuli, J.P., Hauck, S.I. and Hartwig, J.F. (2000) High Turnover Number and Rapid, RoomTemperature Amination of Chloroarenes Using Saturated Carbene Ligands. Organic Letters, 2, 1423-1426. 
http://dx.doi.org/10.1021/ol005751k

[11] Huang, J., Grasa, G. and Nolan, S.P. (1999) General and Efficient Catalytic Amination of Aryl Chlorides Using a Palladium/Bulky Nucleophilic Carbene System. Organic Letters, 1, 1370-1309. http://dx.doi.org/10.1021/ol990987d

[12] Grundeman, S., Albrecht, M., Kovacevic, A., Faller, J.W. and Crabtree, R.H. (2002) Bis-Carbene Complexes from Oxidative Addition of Imidazolium C-H Bonds to Palladium(0). Journal of the Chemical Society, Dalton Transactions, 2163-2167. http://dx.doi.org/10.1039/b110964b

[13] Igau, A., Grutzmacher, H., Baceiredo, A. and Bertrand, G. (1988) Analogous $\alpha, \alpha^{\prime}$-Bis-Carbenoid, Triply Bonded Species: Synthesis of a Stable $\lambda^{3}$-Phosphinocarbene- $\lambda^{5}$-Phosphaacetylene. Journal of the American Chemical Society, 110, 6463-6466. http://dx.doi.org/10.1021/ja00227a028

[14] Caddick, S. and Kofie, W. (2002) Observations on the Intramolecular Heck Reactions of Aromatic Chlorides Using Palladium/Imidazolium Salts. Tetrahedron Letters, 43, 9347-9350. http://dx.doi.org/10.1016/S0040-4039(02)02340-7

[15] Menicagli, R., Malanga, C., Dell’Innocenti, M. and Lardicci, L. (1987) Triisobutylaluminum-Assisted Reductive Rearrangement of Alkyl 1-Alkenyl Acetals: An Easy Synthesis of $\beta$-Alkoxy Alcohols. The Journal of Organic Chemistry, 52, 5700-5704. http://dx.doi.org/10.1021/jo00235a010

[16] Kaiwar, V., Rees, C.B., Gray, E.J. and Neidle, S. (1995) Synthesis of 9-[cis-3-(hydroxymethyl)cyclobutyl]-adenine and -guanine. Journal of the Chemical Society, Perkin Transactions, 2281-2288. http://dx.doi.org/10.1039/p19950002281

[17] Eremeev, A.V., Polyak, F.D., Vosekalna, I.A., Chervin, I.I., Nasibov, S.S. and Kostyanovskii, R.G. (1984) Absolute Configuration of Diastereomeric Derivatives of N-Substituted Aziridine-2-Carboxylic Acids. Chemistry of Heterocyclic Compounds, 20, 1102-1107. http://dx.doi.org/10.1007/BF00503598

[18] Bankston, D., Fang, F., Huie, E. and Xie, S. (1999) Palladium(II) Acetate-Tris(triphenylphosphine)rhodium(I) Chloride: A Novel Catalytic Couple for the Intramolecular Heck Reaction. The Journal of Organic Chemistry, 64, 34613466. http://dx.doi.org/10.1021/jo982058q

[19] Marimoto, K., Hirano, K., Sato, T. and Miura, M. (2011) Synthesis of Isochromene and Related Derivatives by Rhodium-Catalyzed Oxidative Coupling of Benzyl and Allyl Alcohols with Alkynes. The Journal of Organic Chemistry, 76, 9548-9551. http://dx.doi.org/10.1021/jo201923d

[20] Giles, R.G.F., Rickards, R.W. and Senanayake, S.B. (1998) Synthesis of Isochroman-3-Ylacetates and Isochromane$\gamma$-Lactones through Rearrangement of Aryldioxolanylacetates. Journal of the Chemical Society, Perkin Transactions, 3949-3956. http://dx.doi.org/10.1039/a807005i

[21] Young, D.W. (2010) Synthetic Chemistry: An Upfront Investment. Nature Chemical Biology, 6, 174-175. http://dx.doi.org/10.1038/nchembio.325

[22] Terashima, M. and Ishii, M. (1982) A Facile Synthesis of 2-Substituted Benzoxazoles. Synthesis, 1982, 484-485. http://dx.doi.org/10.1055/s-1982-29847

[23] Minami, T., Isonaka, T., Okada, Y. and Ichikawa, J. (1993) Copper(I) Salt-Mediated Arylation of Phosphinyl-Stabilized Carbanions and Synthetic Application to Heterocyclic Compounds. The Journal of Organic Chemistry, 58, 70097015. http://dx.doi.org/10.1021/jo00077a018

[24] Park, H.J., Park, M.S., Lee, T.H. and Park, K.H. (2013) Synthesis of 2-Styrylbenzoxazole Derivatives by the Reaction of Styrylphenolic Schiff Bases with Thianthrene Cation Radical. Journal of Heterocyclic Chemistry, 50, 663-667. http://dx.doi.org/10.1002/jhet.1707 